\title{
Impact of SARS-CoV-2 on Ocular Surface Pathology and Treatment Practices: a Review
}

\author{
Sila Bal ${ }^{1}$ James Chodosh ${ }^{1} \cdot$ Nandini Venkateswaran ${ }^{1,2}$ \\ Accepted: 25 June 2021 / Published online: 5 August 2021 \\ (c) The Author(s), under exclusive licence to Springer Science+Business Media, LLC, part of Springer Nature 2021
}

\begin{abstract}
Purpose of Review The ocular surface is a potential site of ocular involvement by SARS-CoV-2 infection. We performed a review of the literature to understand the pathogenesis of SARS-CoV-2 disease manifestations on the ocular surface as well as to elucidate emerging treatment patterns and practice changes during the COVID-19 pandemic.

Recent Findings The ocular manifestations of SARS-CoV-2 are likely limited to a mild and transient conjunctivitis. Other manifestations have not been validated in larger cohorts. Ocular surface tissue should be considered potentially infectious due to the presence of host receptors on surface tissues. The availability of donor tissue in lower-middle income countries has been greatly impacted by the pandemic and would benefit from further investigation into transmissibility through donor tissue. Summary Transmission of SARS-CoV-2 through the ocular surface has yet to be confirmed. The most common ocular manifestation is a mild conjunctivitis. Ocular surface surgeons face specific challenges in the use of donor tissues and aerosolizing procedures and have adapted practice patterns accordingly.
\end{abstract}

Keywords COVID-19 ocular surface $\cdot$ COVID-19 eye $\cdot$ SARS-CoV-2 ocular surface $\cdot$ Ocular surface pathology COVID-19

\section{Introduction}

Severe Acute Respiratory Syndrome Coronavirus-2 (SARS$\mathrm{CoV}-2$ ) is an enveloped, single plus-stranded RNA virus of the family coronaviridae $[1,2 \bullet, 3,4]$. The virus is known to infect the respiratory tract and can result in respiratory distress, systemic inflammatory responses, and in severe cases, mortality. Like most coronaviruses, SARS-CoV-2 symptoms are not restricted to the respiratory tract and there have been many reports of widespread symptomatology, including ocular involvement. These reports have mainly focused on the

This article is part of the Topical Collection on Ocular Surface

Nandini Venkateswaran

nandini_venkateswaran@meei.harvard.edu

Sila Bal

sila_bal@meei.harvard.edu

James Chodosh

james_chodosh@meei.harvard.edu

1 Massachusetts Eye and Ear, Harvard Medical School, Boston, MA, USA

2 Massachusetts Eye and Ear Infirmary, Waltham, MA, USA ocular surface, with the major reported ocular findings being conjunctivitis and ocular surface irritation.

There are several proposed mechanisms for ocular involvement. Most widely accepted among the ophthalmological community is the theory that the ocular surface represents a site of inoculation for this airborne virus via inoculation of the conjunctiva by droplets. Other plausible mechanisms include migration of the virus from the nasolacrimal duct, hematogenous infection of the lacrimal gland, and/or inoculation of the regional mucosal immune system in the nasal cavity [1, 2•, 3, 4]. Despite these proposed mechanisms, transmission via the ocular route has yet to be definitively established and evidence of expression of an essential host receptor, the angiotensin converting enzyme 2 receptor (ACE2), by the ocular surface epithelium is controversial.

\section{Methods}

We performed a review of the literature on SARS-CoV-2 and the ocular surface in order to understand the pathogenesis behind ocular surface pathology of SARS-CoV-2 as well as emerging treatment patterns and clinical practice 
changes. Our review identified 1046 published reports related to SARS-CoV-2 and the eye. Following title and abstract screening, 67 papers underwent full text review, and of those, 35 were deemed relevant to our review questions. The ocular surface is perhaps the most critical structure in understanding the pathophysiology of ocular involvement of SARS-CoV-2. The effect of the virus on ocular surface treatment guidelines and ophthalmology practice patterns in our communities has been significant and will certainly have an enduring impact.

\section{Ocular Surface Pathology}

There are two important questions regarding SARS-CoV-2 and the ocular surface. First, can an individual become infected through ocular surface exposure to the virus, and second, are the ocular secretions of an individual infected with SARS-CoV-2 infectious to others? Anecdotal evidence has suggested that the ocular surface may serve as a site for infection [5, 6]. However, it remains unclear whether SARS-CoV-2 transmission occurs via the ocular surface and whether ocular secretions of infected individuals are infectious to uninfected individuals.

Penetration of SARS-CoV-2 into the host cell requires two distinct processes. First, the viral spike proteins, which are synthesized in a precursor folded state, must undergo proteolytic cleavage by the serine protease, transmembrane serine protease 2 (TMPRSS2) $[4,7,8]$. Once activated, the spike glycoproteins may then interact with the ACE2 host receptor, facilitating viral envelope/cell membrane fusion and infection of the host cell $[4,7,8]$.

While ACE2 mRNA is expressed in nearly all organs, ACE2 protein expression in ocular tissues remains controversial [2•]. Immunohistochemical analysis of human eye sections stained with anti-ACE2 antibody showed moderate staining in superficial epithelial cells. Similarly, Western blot data has shown expression of TMTPRSS2 protein in corneal epithelial cells [8]. The strongest evidence to support a potential conjunctival route of infection was performed by Hui and colleagues [7]. The authors successfully infected conjunctival mucosa in ex vivo organotypic cultures, supporting the conjunctiva as a route of infection [7]. However, immunohistochemical staining was seen only in the substantia propria. There was no staining in the conjunctival epithelium, suggesting that the conjunctival epithelium itself was resistant to infection. The staining observed in the subepithelial substantia propria may represent replication in vascular endothelial cells or other cells including resident immune cells.

ACE2 expression alone is not enough to infer SARSCoV-2 transmissibility in ocular structures. In one report, there was no evidence of SARS-CoV-2 replication using
RT-PCR in seven independent donor samples.[9]. The results were negative when using plaque assay but showed positive replication of herpes simplex 1 (HSV-1) and weak replication of Zika virus in corneal tissues [9]. It is unlikely that the cornea supports infection of SARSCoV-2. The authors also found Type III interferon expression in corneal tissue and that blockade of Type III interferon and its receptors enhances replication of Zika and HSV-1 but not SARS-CoV-2, indicating that SARS-CoV-2 immunity in the cornea is likely mediated by another pathway [9].

While ACE2 may be expressed on the ocular surface, reports on whether the ocular secretions from an individual infected with SARS-CoV-2 are infectious toward uninfected individuals vary. The likelihood of virus in a sample correlates closely with the amount of viral RNA in the tissue [10]. In all the reports we identified, the amount of SARS-CoV-2 RNA eluted from conjunctival swabs and identified by RT-PCR was considerably lower than from nasopharyngeal swabs [2•,11-15]. While infection through contact with ocular secretions is possible, it is much less likely than being infected by respiratory secretions, whether airborne or by direct contact. The proportion of conjunctival swabs positive for SARS-CoV-2 RNA by RT-PCR in patients with ocular symptoms ranged from 0 to $65 \%[2 \bullet, 11-15]$. The true RT-PCR positivity of tears in those with SARS-CoV-2 is likely closer to the lower estimates reported in the literature, which in one report of pooled sensitivity was around $1 \%[16 \bullet]$.

We found that ocular manifestations of SARS-CoV-2 are uncommon and likely limited to a mild follicular conjunctivitis. Many of the reports on ocular findings in patients with COVID-19 used terms not specific to conjunctivitis, including "congestion"[17] and "chemosis"[17], and were limited to subjective assessments by internal medicine teams viewed retrospectively in medical records. In one retrospective review, 12 of 38 hospitalized patients had "oculamanifestations" [18]. Of these, only three patients had conjunctival hyperemia, which may suggest the diagnosis of conjunctivitis, while the remaining had chemosis, which is not uncommon in hospitalized patients who may experience fluid overload or third spacing. Therefore, many reported ocular manifestations likely represent non-infectious manifestations of systemic illness. There are single cases in the literature of associations between SARS-CoV-2 and other ocular manifestations including hemorrhagic conjunctivitis, [19] epithelial keratitis, [20] and neuro-ophthalmic complications such as optic neuritis or cranial neuropathies [21]. However, these are limited to single case reports. Despite the wide range of reported prevalence of ocular symptoms, we suspect that the true prevalence is likely low, well less than $5 \%$ indicated by meta-analyses that included the aforementioned studies in their calculations [2•, 16•, 22•]. 


\section{Impact on Ocular Surface Treatment}

The impact of SARS-CoV-2 extends beyond the emergency room and intensive care units. Patients with known SARS-CoV-2 have been shown to have RT-PCR positivity in a range of tissues, including the ocular surface as discussed above.

Amniotic membrane is an essential tool in the ocular surface surgeon's armamentarium and is essential in the treatment of many severe ocular surface conditions. Amnion is a donor tissue harvested from the amniotic membrane of donor placentas. Similar to all donor tissue, amnion has the potential to transmit infectious diseases from the host to the donor and reports have shown that viral RNA is present in amnion [23, 24]. However, it remains unclear whether amniotic membrane preparations from infected donors can transmit SARS-CoV-2 via the ocular surface and whether the viral envelope would survive freeze-thaw or acid-alkali washes.

The United States Food and Drug Administration guidance on tissue-based products continues to indicate that there is insufficient evidence supporting transmission through tissue transplantation [25]. Still, major eye banks such as the Eye Bank Association of America (EBAA) and the Global Alliance of Eye Bank Associations (GAEBA) have recommended excluding donors recently infected with SARS-CoV-2 [25-28].

Such conservative exclusion criteria are developed based on the virus's transmissibility, prevalence, and effects [26]. However, with such a high rate of spread, the avoidance of all donor tissue affected by SARS-CoV-2 may be unfeasible.[28]. During the early pandemic, there was a $30 \%$ reduction in corneal donations in the USA [25]. This gap was even greater in lower-middle income countries (LMIC) as travel restrictions, an international shortage in donor supply, and limited accessibility between countries resulted in a severe decline in global corneal donor availability [25]. This shortage the development of innovative alternatives to traditional keratoplasty, including artificial corneas and corneal cell therapies, more critical [27].

Furthermore, donor tissue is usually double disinfected-first, with povidone iodine before removal, followed by Polyvinylpyrrolidone solution prior to storage. This method has been shown to inactivate $99.9 \%$ of viruses within $60 \mathrm{~s}$ and would presumably inactivate any SARSCoV-2 viral activity as well [27].

Given the disproportionate impact of the donor shortage on LMICs, costly innovations such as artificial corneas may be of limited help. Global donor shortages and delays due to regulatory testing may necessitate the need for long-term corneal storage in countries that rely on host countries as a tissue source. These long-term storage techniques include glycerol preservation, lyophilization, and gamma irradiation [29,30]. Glycerol preservation is the most adoptable technique, with reports of donor tissue storage for up to 10 years with clarity similar to fresh tissue $[29,30]$. The major limitation to this technique is that the corneal endothelium becomes unviable [30]. However, in urgent therapeutic cases in regions without access to other donor tissue, long-term glycerol preservation ensures that patients have access to tissue. In one paper, the therapeutic success was $91 \%$ in glycerol preserved corneas versus $87 \%$ in fresh tissue [29]. Glycerol itself has both antimicrobial and anti-protease properties while the resultant acellularity reduces antigenicity and thus limits the need for post-operative immunotherapy with topical corticosteroids [29].

The impact of SARS-CoV-2 is not limited to donor tissues. Blood products, such as autologous serum tears, may represent yet another source of infection. However, there is limited evidence of re-infection in the case of autologous tears harvested during an infective period [31]. Use of autologous tears pose no risk of transmission from another individual [31].

\section{Ophthalmology Practice Patterns}

Prior to the start of the pandemic, ophthalmologists rarely, if ever, used masks. Slit lamp shields, if in use, were small and covered just the area between the mouth of the physician and the patient. Since the start of the pandemic, mask mandates necessitated modifications of examination techniques due to the impact of masking on the ophthalmological exam, including lens fogging, masks obstructing the view, and patient comfort. Furthermore, many practices have adopted larger shields between the physician and the patient. These shields may be clumsy and represent yet another obstacle for a thorough clinical examination. In one study by Ong and colleagues, the authors examined the impact of masks versus shields on large droplet aerosol transmission [32]. They found that slit-lamp shields mainly reduced the risk of large-droplet transmission. However, there was still a risk of infectious aerosols on the side of the shield facing the ophthalmologist, likely due to the minor leaks adjacent to the oculars of the slit lamp [32]. Furthermore, slit lamp shields had a lower efficacy against smaller aerosols and the shields could actually act as fomites for contact transmission if not properly disinfected between every patient. They confirmed that regular face masks provided the most complete protection against both aerosol and droplet transmission [32]. In addition, large slit-lamp shields were unlikely to add any significant additional benefit to the patient or the provider if masks were worn properly by both persons. 
While shields may not serve to add any additional benefit during routine exams, they may serve a purpose in certain aerosol generating procedures unique to the ocular surface, such as refractive surgery. There are several reports on best practices for refractive surgeons [33, 34]. These practices involve horizontal and vertical protocols [34]. Horizontal protection protocols, the protection of transmission between staff and patients, included measures such as mandatory masking, pretreatment surveys, distancing, and proper ventilation [33, 34]. Vertical protection, the protection of patients, pre-, intra-, and post-operatively, included gowning patients to prevent fomite transmission, povidone-iodine ocular surface cleanses preoperatively, and counseling about the importance of eye hygiene during the post-operative period.[33, 34]. Povidone-iodine is of particular interest due to its virucidal properties and ability to inactivate SARSCoV-2 on the ocular surface.[35]. Ocular surfaces washes performed 10 min preoperatively likely eliminate infectious viruses on the surface providing enhanced protection to both the patient and the provider [35]. Many surgeons agreed that refractive surgery could be performed safely during the pandemic, as long as the appropriate personal protective equipment and disinfection procedures are followed. However, enhanced shielding may be necessary in certain high-risk procedures. For example, microkeratome assisted flap creation is associated with greater aerosol dispersion compared to femtosecond lased assisted flap creation and may require additional shielding and physical distancing between the patient and the provider [33].

In many hospitals around the world, elective corneal procedures were canceled during the lockdown period, with one tertiary center for ophthalmic care in the United Kingdom reporting a $92 \%$ reduction in elective corneal surgeries [36]. Strict risk stratification and identification of emergency surgeries resulted in significant delays in the treatment of progressive corneal pathology, such as keratoconus. In many institutions, vision preserving procedures such as collagen crosslinking were postponed, resulting in progression of disease. In one retrospective review of 46 eyes that had crosslinking withheld for an average period of three months, $70 \%$ of eyes showed progression in accordance with the ABCD criteria during the period of delay [37]. As hospitals and communities continue to re-open, a focus should be made toward helping individuals receive the care they need in a timely fashion in order to prevent undue progression of disease.

\section{Conclusion}

The impact of SARS-CoV-2 on the ocular surface is complex. Transmissibility through ocular surface tissues is controversial, and so far lacks confirmation. However, given the evidence for host receptors on the ocular surface, ocular secretions of individuals with SARS-CoV-2 should still be considered potentially infectious. Although rare, our review found that there are ocular manifestations of SARS-CoV-2, most commonly a mild and transient conjunctivitis.

Ocular surface surgeons face unique challenges related to donor products, including corneal tissue, amnion, and autologous serum tears. Donor corneal tissue represents an area of significant concern given the disproportionate impact on LMICs due to decreased host availability, transportation limitations, and increased wait times. Long-term storage techniques may serve as a potential temporary solution to tissue limitations and may represent a viable option for recipient countries.

Refractive surgery techniques associated with increased risk of aerosol generation may benefit from larger shields and greater distancing between the patient and the provider. Other ophthalmology infection control measures that were adopted early in the pandemic, such as extensive slit-lamp shields, may not provide any additional benefit to simple mask-wearing. Given the recent change in mask mandates, physicians may choose to consider continuing to mask patients during the close-contact slit-lamp examination.

Ultimately, SARS-CoV-2 has had a significant impact on ocular surface treatment and practices. The impact of the COVID-19 pandemic on the availability of corneal donor tissue in LMICs is particularly concerning, given that it may extend far beyond the time bounds of the acute phase of this public health crisis. Looking forward, ocular surface surgeons should use the lessons of this pandemic to continue to advocate for solutions to address the disproportionate access to donor tissue.

\section{Declarations}

Conflict of Interest The authors declare no conflict of interest.

Human and Animal Rights This article does not contain any studies with human or animal subjects performed by any of the authors.

\section{References}

Papers of particular interest, published recently, have been highlighted as:• Of importance

1. Seah I, Agrawal R. Can the coronavirus disease 2019 (COVID19) affect the eyes? A review of coronaviruses and ocular implications in humans and animals. Ocul Immunol Inflamm. 2020;28(3 PG-391-395):391-5.

2. Nora RL, Putera I, Khalisha DF, Septiana I, Ridwan AS, Sitompul R. Are eyes the windows to COVID-19? Systematic review 
and meta-analysis. BMJ Open Ophthalmol. 2020;5(1 PG-13):13. This meta-analysis on the ocular manifestations of COVID19 included 14 studies, including a pooled analysis of PCR positivity in tears. This provides unique insight into the true prevalence of ocular manifestations, $5 \%$ in this report, and PCR positivity in individuals with ocular symptoms, $38 \%$.

3. Chen X, Yu H, Mei T, Chen B, Chen L, Li S, Zhang X, Sun X. SARS-CoV-2 on the ocular surface: Is it truly a novel transmission route? Br J Ophthalmol. 2020. https://doi.org/10.1136/bjoph thalmol-2020-316263.

4. Barnett BP, Wahlin K, Krawczyk M, Spencer D, Welsbie D, Afshari N, et al. Potential of Ocular Transmission of SARSCoV-2: A Review. Vis. 2020;4(3):40.

5. Fieldstadt E. Virologist hospitalized with coronavirus believes he got it through his eyes. 2020. Available from: https://www.nbcne ws.com/news/us-news/virologist-hospitalized-coronavirus-belie ves-he-got-it-through-his-eyes-n1206956. Accessed 1 Feb 2021.

6. Yan A. Chinese expert who came down with Wuhan coronavirus after saying it was controllable thinks he was infected through his eyes. 2020. Available from: https://www.scmp.com/news/ china/article/3047394/chinese-expert-who-came-down-wuhancoronavirus-after-saying-it-was. Accessed 22 Feb 2021.

7. Hui KPY, Cheung MC, Perera R, Ng KC, Bui CHT, Ho JCW, et al. Tropism, replication competence, and innate immune responses of the coronavirus SARS-CoV-2 in human respiratory tract and conjunctiva: an analysis in ex-vivo and in-vitro cultures. Lancet Respir Med. 2020;8(7 PG-687-695):687-95.

8. Zhou L, Xu Z, Castiglione GM, Soiberman US, Eberhart CG, Duh EJ. ACE2 and TMPRSS2 are expressed on the human ocular surface, suggesting susceptibility to SARS-CoV-2 infection. Ocul Surf. 2020;18(4):537-44.

9. Miner JJ, Platt DJ, Ghaznavi CM, Chandra P, Santeford A, Menos AM, et al. HSV-1 and zika virus but not SARS-CoV-2 replicate in the human cornea and are restricted by corneal type III interferon. Cell Rep. 2020;33(5):108339.

10. Singanayagam A, Patel M, Charlett A, Lopez Bernal J, Saliba V, Ellis J, Ladhani S, Zambon M, Gopal R. Duration of infectiousness and correlation with RT-PCR cycle threshold values in cases of COVID-19, England, January to May 2020. Euro Surveill. 2020;25(32):2001483. https://doi.org/10.2807/15607917.ES.2020.25.32.2001483.

11. Cavalleri M, Brambati M, Starace V, Capone L, Nadin F, Pederzolli M, et al. Ocular features and associated systemic findings in SARS-CoV-2 infection. Ocul Immunol Inflamm. 2020;28(6 PG-916-921):916-21.

12. Li X, Chan JF, Li KK, Tso EY, Yip CC, Sridhar S, Chung TW, Chiu KH, Hung DL, Wu AK, Chau SK, Liu R, Lung KC, Tam AR, Cheng VC, To KK, Chan KH, Hung IF, Yuen KY. Detection of SARS-CoV-2 in conjunctival secretions from patients without ocular symptoms. Infection. 2021;49(2):257-65. https://doi.org/ 10.1007/s15010-020-01524-2.

13 Ho D, Low R, Tong L, Gupta V, Veeraraghavan A, Agrawal R. COVID-19 and the ocular surface: a review of transmission and manifestations. Ocul Immunol Inflamm. 2020;28(5 PG-726-734):726-34

14. Atum M, Boz AAE, Çakır B, Karabay O, Köroğlu M, Ögütlü A, et al. Evaluation of conjunctival swab PCR results in patients with SARS-CoV-2 infection. Ocul Immunol Inflamm. 2020;28(5 PG-745-748):745-8.

15. Shemer A, Einan-Lifshitz A, Itah A, Dubinsky-Pertzov B, Pras E, Hecht I. Ocular involvement in coronavirus disease 2019 (COVID-19): A clinical and molecular analysis. Int Ophthalmol. 2021;41(2):433-40. https://doi.org/10.1007/ s10792-020-01592-1.

16. Ulhaq ZS, Soraya GV. The prevalence of ophthalmic manifestations in COVID-19 and the diagnostic value of ocular tissue/ fluid. Graefes Arch Clin Exp Ophthalmol. 2020;258(6 PG-13511352):1351-2. This meta-analysis of seven studies identified a large range in reported ocular manifestations of COVID-19, from $2 \%$ to $32 \%$, highlighting the need for further investigation into the true ocular manifestations of SARS-CoV-2 infection.

17. Liu WR, Zuo GJ, Qin Y. Clinical characteristics and outcomes of 2019-nCoV-infected patients admitted at different time periods. Eur Rev Med Pharmacol Sci. 2020;24(14 PG-7826-7833):7826-33.

18. Wu P, Duan F, Luo C, Liu Q, Qu X, Liang L, et al. Characteristics of ocular findings of patients with coronavirus disease 2019 (COVID-19) in Hubei Province. China JAMA Ophthalmol. 2020;138(5 PG-575-578):575-8.

19 Navel V, Chiambaretta F, Dutheil F. Haemorrhagic conjunctivitis with pseudomembranous related to SARS-CoV-2. Am J Ophthalmol Case Rep. 2020;19:100735.

20 Cheema M, Aghazadeh H, Nazarali S, Ting A, Hodges J, McFarlane A, et al. Keratoconjunctivitis as the initial medical presentation of the novel coronavirus disease 2019 (COVID-19). Can J Ophthalmol. 2020;55:125-9.

21 Tisdale AK, Chwalisz BK. Neuro-ophthalmic manifestations of coronavirus disease 19. Curr Opin Ophthalmol. 2020;31(6 PG-489-494):489-94.

22.• Grant MC, Geoghegan L, Arbyn M, Mohammed Z, McGuinness L, Clarke EL, et al. The prevalence of symptoms in 24,410 adults infected by the novel coronavirus (SARS-CoV-2; COVID-19): a systematic review and meta-analysis of 148 studies from 9 countries. PLoS One. 2020;15(6):e0234765. This large meta-analysis comprised of 24,410 adults with COVID-19 reported that the prevalence of conjunctivitis in patients with COVID-19 infection is likely $2 \%$ or less.

23. Penfield CA, Brubaker SG, Limaye MA, Lighter J, Ratner AJ, Thomas KM, et al. Detection of severe acute respiratory syndrome coronavirus 2 in placental and fetal membrane samples. Am J Obstet Gynecol MFM. 2020;2(3):100133.

24 Zamaniyan M, Ebadi A, Aghajanpoor S, Rahmani Z, Haghshenas M, Azizi S. Preterm delivery, maternal death, and vertical transmission in a pregnant woman with COVID-19 infection. Prenat Diagn. 2020;40:1759-61.

25 AlMutlak M, Li JY, Bin Helayel H, Fairaq R. The future of corneal donation and transplantation; insights from Covid-19 pandemic. Cornea. 2020;40(3):274-6.

26. Desautels JD, Moshirfar M, Martheswaran T, Shmunes KM, Ronquillo YC. Risks posed to corneal transplant recipients by COVID-19-affected donors. Ophthalmol Ther. 2020;9(3 PG-371-379):371-9.

27. Ang M, Moriyama A, Colby K, Sutton G, Liang L, Sharma N, Hjortdal J, Shun Chiu Lam D, Williams GP, Armitage J, Mehta JS. Corneal transplantation in the aftermath of the COVID-19 pandemic: An international perspective. Br J Ophthalmol. 2020;104(11):1477-81. https://doi.org/10.1136/bjoph thalmol-2020-317013.

28. Aldave AJ, DeMatteo J, Chamberlain WD, Philippy B, Farooq AV, Buckman N, et al. COVID and the Cornea: From Controversies to Consensus: Report of the Eye Bank Association of America Medical Advisory Board Policy and Position Review Subcommittee. Cornea. 2021;40(7):809-16.

29 Gupta N, Dhasmana R, Maitreya A, Badahur H. Glycerol-preserved corneal tissue in emergency corneal transplantation: an alternative for fresh corneal tissue in COVID-19 crisis. Indian J Ophthalmol. 2020;68(7 PG-1412-1416):1412-6.

30 Chaurasia S, Das S, Roy A. A review of long-term corneal preservation techniques: Relevance and renewed interests in the COVID-19 era. Indian J Ophthalmol. 2020;68(7 PG-1357-1363):1357-63. 
31. Giannaccare G, Buzzi M, Versura P. Use of eye drops obtained from homologous blood source in the time of COVID-19: is there cause for ophthalmological concern? Blood Transfus. 2020;18(3 PG-233-234):233-4.

32 Ong SC, Razali MAB, Shaffiee L, Yap JX, Fei TTY, Loon $\mathrm{SC}$, et al. Do slit-lamp shields and face masks protect ophthalmologists amidst COVID-19? Ophthalmology. 2020;127(10 PG-1427-1429):1427-9.

33. Zhang J, Aslanides IM, Selimis V, Lu NJ, Liu WJ, Jiang $\mathrm{HX}$, et al. A comprehensive strategy for laser corneal refractive surgery during the COVID-19 epidemic in a Tertiary Teaching Hospital in Wenzhou. China J Ophthalmol. 2020;2020(PG-4835630):4835630.

34. Sharma N, Khamar P, Sachdev MS, Maheshwari S, Gupta K, Sinha R, et al. All India Ophthalmological Society - Preferred practice in refractive surgery during the COVID-19 pandemic. Indian J Ophthalmol. 2020;68(7 PG-1263-1268):1263-8.
35. Kariwa H, Fujii N, Takashima I. Inactivation of SARS coronavirus by means of povidone-iodine, physical conditions and chemical reagents. Dermatology. 2006;212 Suppl(Suppl 1):119-23.

36. Din N, Phylactou M, Fajardo-Sanchez J, Watson M, Ahmad S. The impact of COVID-19 on acute and elective corneal surgery at Moorfields Eye Hospital London. Clin Ophthalmol. 2021;15:1639-45.

37. Shah H, Pagano L, Vakharia A, Coco G, Gadhvi KA, Kaye SB, Romano V. Impact of COVID-19 on keratoconus patients waiting for corneal cross linking. Eur J Ophthalmol. 2021. https:// doi.org/10.1177/11206721211001315.

Publisher's Note Springer Nature remains neutral with regard to jurisdictional claims in published maps and institutional affiliations. 